\title{
THE IMPLICATIONS OF THE TADOMA METHOD OF SPEECHREADING FOR SPOKEN LANGUAGE PROCESSING
}

\author{
Charlotte M. Reed \\ Research Laboratory of Electronics \\ Massachusetts Institute of Technology \\ Cambridge, MA 02139
}

\begin{abstract}
Strong support of the capacity of touch as a communicative sense is provided by the Tadoma method of communication. Through this method, individuals who are deaf-blind have been able to acquire a full range of spoken language abilities. In the Tadoma method, direct contact is made between the hand of the deaf-blind receiver and the face of a talker to monitor the various articulatory actions that occur during speech. Studies conducted with a group of experienced deaf-blind practitioners of Tadoma have documented their abilities for speech reception, speech production, and linguistic competence. The results of this research indicate that individuals who suffered deaf-blindness in early childhood (e.g., around 18 months of age) can understand speech produced at slow-to-normal rates with reasonable accuracy, can produce speech that is reasonably intelligible to many listeners, and have an extensive command of English that compares favorably in many areas to that of hearing individuals. The performance of these deaf-blind individuals implies the adequacy of the tactual sense to support the development of speech and language and thereby provides a strong impetus for continued research on the development of sensory-substitution devices for spoken language processing. Current efforts on the development and evaluation of artificial tactile devices for speech communication will be discussed.
\end{abstract}

\section{INTRODUCTION}

The capacity of the tactual sense to support the development of speech and language has been the subject of much debate. The issues that have been argued include the channel capacity of the tactual compared to the auditory sense and the exclusivity of the speech communication process to the sense of hearing. A convincing existence proof of the potential of the tactual sense for communication is available and documented in the abilities of experienced deaf-blind users of the Tadoma method of speechreading.

\section{BRIEF DESCRIPTION AND HISTORY OF THE TADOMA METHOD}

The Tadoma method of speechreading is based on vibrotactile reception of the articulatory movements and actions that occur during the production of speech. The Tadoma method was developed by educators to provide children who were both deaf and blind with access to speech and language [e.g., see 1,2,3]. In this method, the hand of the deaf-blind receiver is placed over the face and neck of the talker such that the thumb rests lightly on the lips and the fingers fan out over the cheek and neck. Methods of instruction were developed for teaching children both to receive and to produce speech. The most active period of instruction through the Tadoma method in programs for the deaf-blind occurred in the years between 1930 and 1960, primarily for a population of children who had become simultaneously deaf/blind as a result of meningitis.

A number of children who received instruction in the Tadoma method at schools for the deaf-blind in various locations around the country eventually became highly skilled in the use of this method [4]. On the basis of their ability to receive speech and language through tactual input alone, these individuals afford researchers the opportunity to explore the capacity of the sense of touch for communication. A series of analytic studies exploring speech reception, speech production, and linguistic ability through Tadoma has been conducted in cooperation with a small group of experienced deaf-blind users of the Tadoma method [e.g., 5,6,7,8,9,10,]. This paper will focus on summarizing results obtained in studies of speech reception with Tadoma users and in discussing the implications of these findings for the reception of speech and language through an alternative modality.

\section{SUMMARY OF ANALYTIC STUDIES}

\subsection{Subjects}

A total of nine deaf-blind individuals who received training in the Tadoma method and were currently using this method as a means of communication participated in the research [see 7]. Three of these individuals took part in an in-depth series of laboratory evaluations over a period of several years, while the remaining six subjects were tested on a subset of the in-depth tests designed as a survey. Illustrative results are presented here for the three in-depth subjects, on whom a comprehensive set of measures is available. Each of these three subjects (LD, RB, and JC) became simultaneously deaf and blind as a result of meningitis with age at onset of 1.5, 1.8, and 7 years, respectively. Age at time of testing was 54, 48, and 53 years for $\mathrm{LD}$, $\mathrm{RB}$, and JC, respectively. Clinical testing of visual and auditory function in these individuals indicates no measurable visual acuity and no audiometric response (except for some response to high-intensity, low-frequency signals, most likely arising from vibrotactile stimulation, for $\mathrm{LD}$ and $\mathrm{RB}$ ). [To ensure that no auditory or visual cues were available during speech testing, subjects were blindfolded and exposed to masking noise.] 


\begin{tabular}{|c|c|c|c|c|c|c|c|}
\hline \multirow[b]{2}{*}{ Subject } & \multicolumn{2}{|c|}{ Isolated Syllables } & \multicolumn{2}{|c|}{ Isolated Words } & \multicolumn{2}{|c|}{ Sentences } & \multirow{2}{*}{$\begin{array}{l}\text { Connected-Discourse } \\
\text { Tracking } \\
\end{array}$} \\
\hline & 24 Consonants & 15 Vowels & Closed Se & Open Set & CID & IEEE & \\
\hline LD & $63 \%$ & $58 \%$ & $92 \%$ & $49 \%$ & $84 \%$ & $34 \%$ & 31 words/min \\
\hline $\mathrm{RB}$ & $58 \%$ & $46 \%$ & $72 \%$ & $34 \%$ & $85 \%$ & $47 \%$ & 30 words/min \\
\hline $\mathrm{JC}$ & $68 \%$ & $65 \%$ & $90 \%$ & $48 \%$ & $83 \%$ & $68 \%$ & 36 words/min \\
\hline
\end{tabular}

Table 1: Summary of speech reception measures with three Tadoma subjects. First column shows scores obtained in nonsense syllable tests using a set of 24 consonants in C-/a/ context and 15 vowels in /h/-V-/d/ context. Second column shows scores on isolated monosyllabic words for the five-alternative Modified Rhyme Test [11] and for phonetically balanced W-22 words [12]. Third column shows percentage correct identification of key words in lists of conversational CID sentences [13] and phonetically balanced IEEE [14] sentences for speaking rates of roughly 2.5 syllables/sec. Fourth column provides continuous-discourse tracking rate [15] in words/min.

\subsection{Speech-Reception Results}

The ability of Tadoma users to understand speech was examined for a variety of materials ranging from nonsense syllables to connected speech. In these studies, the Tadoma user placed his/her hand on the face and neck of the talker administering the test. For closed-response tests, the subject was provided with a list of the response alternatives in Braille and was asked to scan the list and select a response following each stimulus presentation. For open-response tests, the subject provided type-written or oral responses (which were clarified when necessary through spelling or fingerspelling).

Performance on a variety of speech tests is summarized in Table 1 for subjects $\mathrm{LD}, \mathrm{RB}$, and $\mathrm{JC}$.

At the basic segmental level, large sets of consonants or vowels are identified at a level of roughly $60 \%$ correct. Analyses of segmental errors indicate highly structured patterns of confusion that are quite similar across subjects. For consonants, confusions were concentrated on errors related to place of articulation within a given manner of production and also included confusions across several classes of production (e.g., affricates and fricatives, fricatives and semivowels); voicing confusions were rarely observed. Although vowel confusions were somewhat more broadly distributed than consonant confusions, three or four major clusters of errors were observed and related to misidentification of the properties of high-low and front-back positioning of the body of the tongue.

The role of linguistic context in the speech-reception abilities of these subjects is demonstrated by their scores on meaningful speech materials. The recognition rate for open-set words in isolation, for example, exceeds predictions based on performance on nonsensesyllables, but underestimates performance obtained on the reception of words in conversational sentences. These results imply that in openset recognition of isolated words the subjects were able to reject nonmeaningful responses through their semantic knowledge, and furthermore were able to exploit their knowledge of syntactical constraints in understanding words in conversational sentences. The superior performance observed on the CID compared to the IEEE sentences reflects differences in the amount of contextual information supplied by the two types of materials.
Such results are similar to those obtained in studies of the effects of speech-to-noise ratio on the auditory reception of speech materials as a function of context. A comparison of performance of Tadoma users on materials from the SPIN test [16] with that of normal-hearing listeners as a function of speech-to-noise ratio is presented in Fig. 1. In this test, the subject's task is to identify the final word in short sentences where the word is presented in either a high predictability (HP) or low predictability (LP) context. In Fig. 1, scores on HP and LP words reported by Kalikow et al. [16] for a group of older listeners (aged 60-75 yrs) are plotted as a function of speech-to-noise ratio. The scores achieved by each of the three Tadoma users are superimposed on the auditory data such that their HP scores are plotted on the HP

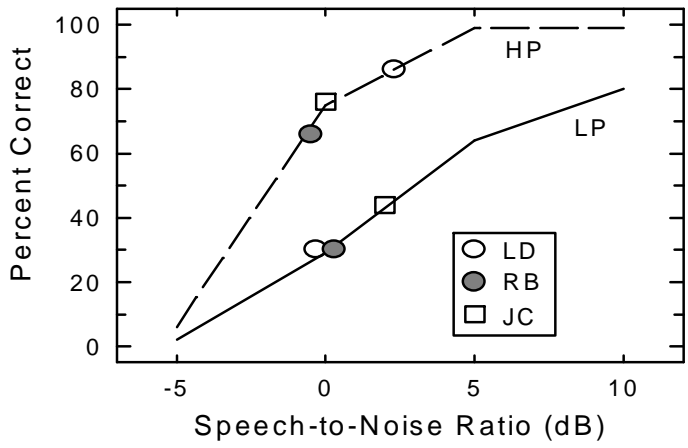

Figure 1. SPIN test results.

curve and their LP scores on the LP curve. The Tadoma scores range between $66-86 \%$ correct for HP words and between $30-44 \%$ for LP words. These scores correspond to performance achieved by the listeners at speech-to-noise ratios in the range of roughly $0-5 \mathrm{~dB}$. Additionally, the difference between HP and LP scores observed for the Tadoma users (32-56 percentage points) falls in the range of the maximal context effects observed for listeners in noise.

The effect of speaking rate on sentence intelligibility was examined by asking talkers to vary their subjective rate of production from slow to fast across different lists of sentences. A range of speaking rates from 
roughly 2-8 syllables/sec (where 5 syllables/sec is representative of normal speaking rate) was produced by the talkers administering these tests. In Fig. 2, the percent-correct reception of key words in lists of CID sentences is plotted as a function of speaking rate in syllables/sec for each of the three subjects. Performance falls off at rates above roughly 3-4 syllables/sec for RB and JC and 6 syllables/sec for LD. Across the sample of nine subjects tested in the survey study, however, maximal performance is typically obtained at rates roughly half those of the normal speaking rate [7].

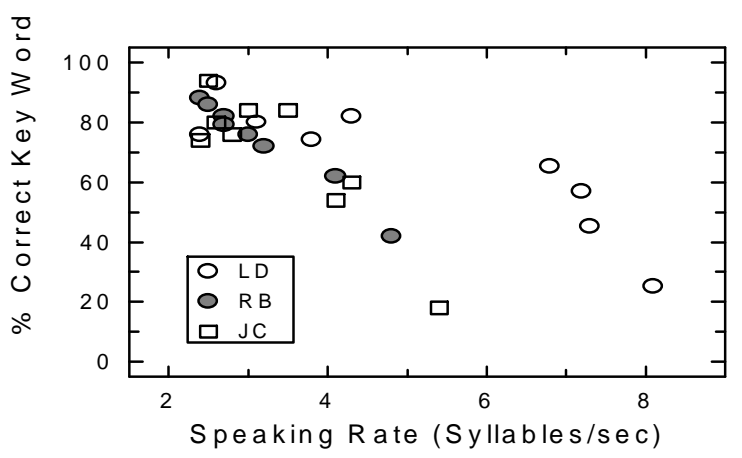

Figure 2. CID key-word reception as a function of rate.

The continuous-discourse tracking procedure [15] was employed to provide a practical estimate of the communication rates achieved by pairs of talkers and Tadoma users. For auditory reception of speech under normal conditions, tracking rate is roughly 100 words $/ \mathrm{min}$. The tracking rates attained by the Tadoma users (30-36 words/min) are roughly one-third as fast as the rates achieved in normal auditory reception of speech. These rates are limited primarily by the time spent correcting errors and secondarily by the slower speaking rates adopted by the talkers and the slower speaking rates employed by the Tadoma users [10].

\section{HOW DOES THE TADOMA USER PROCESS SPEECH?}

The reception of speech through Tadoma is based entirely on information concerning the various movements and actions that take place during articulation and that can be felt through the placement of the hand on the face and neck. The primary cues available to the Tadoma user include the up-down and in-out movements of the lips, movements of the jaw, airflow at the lips, and vibration on the neck. Insight into the relation between these cues and the reception of information concerning consonant and vowel segments has been derived from studies of segmental confusions made both by experienced Tadoma users [6,7,8] and laboratory-trained subjects [17] and from experiments conducted with an artificial Tadoma system $[18,19]$. For example, consonant voicing appears to be cued primarily by vibration that can be felt on both the neck and jaw and secondarily by airflow characterisitcs, while different manners of articulation are distinguished by differences in the intensity and concentration of airflow at the lips. The most salient information for vowels appears to be provided by in-out and up-down lip movements for determining roundedness and lip separation and by jaw movements for distinguishing vowels in which the body of the tongue is lowered. These cues appear to be sufficient for transmitting roughly 3 bits of information for consonants and 2 bits for vowels [6]. Incomplete segmental information appears to be combined with semantic and syntactic knowledge, leading to the ability to receive contextual messages with a reasonable degree of accuracy.

The uniqueness of successful Tadoma users lies in their ability to process continuous streams of tactual stimuli in such a way as to derive meaning from these patterns. While all the Tadoma users surveyed exhibited very similar performance at the segmental level, the distinguishing characteristic of the more successful users appears to lie in their ability to exploit the use of contextual cues to decode spoken messages [7]. Similarly, studies with naive laboratory subjects indicated that while they could be trained to identify speech segments with 50-100 hrs of practice [17], the goal of conversational speech reception comparable to that of experienced Tadoma users was not attained within 500-600 hrs of practice.

\section{IMPLICATIONS OF TADOMA FOR SENSORY SUBSTITUTION IN SPEECH COMMUNICATION}

The speech-reception abilities of the Tadoma users documented above demonstrate the capability of the tactual sense for supporting speech and language processing. For subject JC, for whom speech and language was already well-established at onset of deaf-blindness, the results presented here indicate her ability to substitute a tactual representation of the speech code for the auditory code with which she was previously familiar. For subjects LD and RB, the implications are somewhat more far-reaching. For these two individuals, the normal process for acquiring speech and language was interrupted in the early stages. Thus, their ability to receive speech and make use of contextual information requiring sophisticated linguistic knowledge indicates that tactual input served to establish a language base as well as to provide access to speech.

Careful scrutiny of the properties of the Tadoma method that account for its success may aid in the advancement of research on artificial tactual displays for speech communication. Of the various methods of encoding and displaying the speech signal to the tactual sense that have been studied in the development of artifical devices [e.g., see 20 and 21], none has resulted in performance through the tactual system alone comparable to that achieved through the Tadoma method. A combination of various factors may contribute to the success of the Tadoma method [discussed in 7 and 21, e.g.]. First, skilled users of this method have received intensive, long-term training and have employed the method over extended periods of time. Second, the multidimensional aspects of the speech display available through Tadoma appear to satisfy various principles of information theory for optimizing information transfer. Third, the use of the hand for reception of tactual stimulation may constitute an advantage from two points of view: the high density of innervation of tactual receptors and the potential engagement of both the cutaneous and kinesthetic components of the tactual sense. Finally, access to the articulators may constitute an advantage, especially in the view of proponents of a motor theory of speech perception. 
The current challenge in research on artificial tactual systems is the development of encoding and display schemes that will permit the reception of continuous speech at information-transfer rates at least as fast as those observed through Tadoma. Recent research has focused on improving information transfer through the development of tactual displays that engage the kinesthetic as well as the cutaneous component of that sensory system [see 22 and 23]. In a recent study exploring the

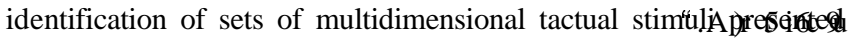
through a display capable of stimulation along the kinestheticcutaneous continuum, Tan [23] estimated information-transfer rates to be roughly $12 \mathrm{bits} / \mathrm{sec}$ (similar to that estimated for communication through Tadoma). Performance through the Tadoma method provides a standard for evaluating the performance of artificial tactual systems, as well as serving to demonstrate the potential of the tactual sense for the communication of speech and language.

\section{ACKNOWLEDGMENTS}

Work supported by research grant number 2 R01 DC 00126 from the National Institute on Deafness and Other Communication Disorders, National Institutes of Health.

\section{REFERENCES}

1. Alcorn, S. (1932). "The Tadoma Method," Volta Rev. 34: 195-198.

2. Stenquist, G. (1974). The Story of Leonard Dowdy: DeafBlindness Acquired in Infancy. Watertown, MA: Perkins School for the Blind.

3. Vivian, R. (1966). "The Tadoma Method: A Tactual Approach to Speech and Speech Reading," Volta Rev. 68: 733-737.

4. Schultz, M.C., Norton, S.J., Conway-Fithian, S., and Reed, C.M. (1984). "A Survey of the Use of the Tadoma Method in the United States and Canada," Volta Rev. 86: 282-292.

5. Norton, S.J., Schultz, M.C., Reed, C.M., Braida, L.D., Durlach, N.I., Rabinowitz, W.M., and Chomsky, C. (1977). "Analytic Study of the Tadoma Method: Background and Preliminary Results," J. Speech Hearing Res. 20: 574-595.

6. Reed, C.M., Durlach, N.I., Braida, L.D., and Schultz, M.C. (1982). "Analytic Study of the Tadoma Method: Identification of Consonants and Vowels by an Experienced Tadoma User," J. Speech Hearing Res. 25: 108-116.

7. Reed, C.M., Rabinowitz, W.M., Durlach, N.I., Braida, L.D., Conway-Fithian, S., and Schultz, M.C. (1985). "Research on theTadoma Method of Speech Communication," J. Acoust. Soc. Am. 77: 247-257.

8. Reed, C.M., Durlach, N.I., Braida, L.D., and Schultz, M.C. (1989). "Analytic Study of the Tadoma method: Effects of Hand Position on Segmental Speech Perception," J. Speech Hearing Res. 32: 921-929.

9. Chomsky, C. (1986). "Analytic Study of the Tadoma Method:
Language Abilities of Three Deaf-Blind Subjects," J. Speech Hearing Res. 29: 332-347.

10. Tamir, T.J. (1989). Characterization of the Speech of Tadoma Users. B.S. Thesis, MIT, Cambridge, MA.

11. House, A.S., Williams, C.E., Hecker, M.H.L., and Kryter, K.D. al $\mathrm{t}$ i o $\mathrm{n}$ T e s t in g M thods: C o n s o n a n t a 1 Differentiation with a Closed-Response Test," J. Acoust. Soc. Am. 37: $158-166$.

12. Hirsh, I.J., Davis, H., Silverman, S.R., Reynolds, E., Eldert, E., and Benson, R.W. (1952). "Development of Materials for Speech Audiometry," J. Speech Hearing Dis. 17: 321-337.

13. Davis, H., and Silverman, S.R. (1970). Hearing and Deafness. New York: Holt, Rhinehart, and Winston.

14. IEEE (1969). "Recommended Practice for Speech Quality Measurements," IEEE Transactions on Audio Electroacoustics 17: 225-246.

15. De Filippo, C.L., and Scott, B.L. (1978). "A Method for Training and Evaluating the Reception of Ongoing Speech.," J. Acoust. Soc. Am. 63: 1186-1192.

16. Kalikow, D.N., Stevens, K.N, and Elliott, L.L. (1977). "Development of Test of Speech Intelligibility in Noise using Sentence Materials with Controlled Word Predictability," J. Acoust. Soc. Am. 61: 1337-1351.

17. Reed, C.M., Doherty, M.J., Braida, L.D., and Durlach, N.I. (1982). "Analytic Study of the Tadoma Method: Further Experiments with Inexperienced Observers," J. Speech Hearing Res. 25: 216-223.

18. Leotta, D.F., Rabinowitz, W.M., Reed, C.M., and Durlach, N.I. (1988). "Preliminary Results of Speech-Reception Tests Obtained with the Synthetic Tadoma System," J. Rehab. Res. 25: 45-52.

19. Henderson, D.R. (1989). Tactile Speech Reception: Development and Evaluation of an Improved Synthetic Tadoma System. M.S. Thesis, MIT, Cambridge, MA.

20. Reed, C.M., Durlach, N.I., Delhorne, L.A., Rabinowitz, W.M., and Grant, K.W. (1989). "Research on Tactual Communication of Speech: Ideas, Issues, and Findings," Volta Rev. 91: 65-78.

21. Bernstein, L.E. (1992). "The Evaluation of Tactile Aids." In I.R. Summers (Ed.) Tactile Aids for the Hearing Impaired (pp. 167186). London: Whurr Publishers.

22. Eberhardt, S.P., Bernstein, L.E., Barac-Cikoja, D., Coulter, D.C., and Jordan, J. (1994). "Inducing Dynamic Haptic Perception by the Hand: System Description and Some Results," Proc. Am. Soc. Mech. Eng. 55: 345-351.

23. Tan, H.Z. (1996). Information Transmission with a Multi-Finger Tactual Display. Ph.D. Thesis, MIT, Cambridge, MA. 


\title{
THE IMPLICATIONS OF THE TADOMA METHOD OF SPEECHREADING FOR SPOKEN LANGUAGE PROCESSING
}

\author{
Charlotte M. Reed \\ Research Laboratory of Electronics \\ Massachusetts Institute of Technology \\ Cambridge, MA 02139
}

\begin{abstract}
Strong support of the capacity of touch as a communicative sense is provided by the Tadoma method of communication. Through this method, individuals who are deaf-blind have been able to acquire a full range of spoken language abilities. In the Tadoma method, direct contact is made between the hand of the deaf-blind receiver and the face of a talker to monitor the various articulatory actions that occur during speech. Studies conducted with a group of experienced deaf-blind practitioners of Tadoma have documented their abilities for speech reception, speech production, and linguistic competence. The results of this research indicate that individuals who suffered deaf-blindness in early childhood (e.g., around 18 months of age) can understand speech produced at slow-to-normal rates with reasonable accuracy, can produce speech that is reasonably intelligible to many listeners, and have an extensive command of English that compares favorably in many areas to that of hearing individuals. The performance of these deaf-blind individuals implies the adequacy of the tactual sense to support the development of speech and language and thereby provides a strong impetus for continued research on the development of sensory-substitution devices for spoken language processing. Current efforts on the development and evaluation of artificial tactile devices for speech communication will be discussed.
\end{abstract}

\section{INTRODUCTION}

The capacity of the tactual sense to support the development of speech and language has been the subject of much debate. The issues that have been argued include the channel capacity of the tactual compared to the auditory sense and the exclusivity of the speech communication process to the sense of hearing. A convincing existence proof of the potential of the tactual sense for communication is available and documented in the abilities of experienced deaf-blind users of the Tadoma method of speechreading.

\section{BRIEF DESCRIPTION AND HISTORY OF THE TADOMA METHOD}

The Tadoma method of speechreading is based on vibrotactile reception of the articulatory movements and actions that occur during the production of speech. The Tadoma method was developed by educators to provide children who were both deaf and blind with access to speech and language [e.g., see 1,2,3]. In this method, the hand of the deaf-blind receiver is placed over the face and neck of the talker such that the thumb rests lightly on the lips and the fingers fan out over the cheek and neck. Methods of instruction were developed for teaching children both to receive and to produce speech. The most active period of instruction through the Tadoma method in programs for the deaf-blind occurred in the years between 1930 and 1960, primarily for a population of children who had become simultaneously deaf/blind as a result of meningitis.

A number of children who received instruction in the Tadoma method at schools for the deaf-blind in various locations around the country eventually became highly skilled in the use of this method [4]. On the basis of their ability to receive speech and language through tactual input alone, these individuals afford researchers the opportunity to explore the capacity of the sense of touch for communication. A series of analytic studies exploring speech reception, speech production, and linguistic ability through Tadoma has been conducted in cooperation with a small group of experienced deaf-blind users of the Tadoma method [e.g., 5,6,7,8,9,10,]. This paper will focus on summarizing results obtained in studies of speech reception with Tadoma users and in discussing the implications of these findings for the reception of speech and language through an alternative modality.

\section{SUMMARY OF ANALYTIC STUDIES}

\subsection{Subjects}

A total of nine deaf-blind individuals who received training in the Tadoma method and were currently using this method as a means of communication participated in the research [see 7]. Three of these individuals took part in an in-depth series of laboratory evaluations over a period of several years, while the remaining six subjects were tested on a subset of the in-depth tests designed as a survey. Illustrative results are presented here for the three in-depth subjects, on whom a comprehensive set of measures is available. Each of these three subjects (LD, RB, and JC) became simultaneously deaf and blind as a result of meningitis with age at onset of 1.5, 1.8, and 7 years, respectively. Age at time of testing was 54, 48, and 53 years for $\mathrm{LD}$, $\mathrm{RB}$, and JC, respectively. Clinical testing of visual and auditory function in these individuals indicates no measurable visual acuity and no audiometric response (except for some response to high-intensity, low-frequency signals, most likely arising from vibrotactile stimulation, for $\mathrm{LD}$ and $\mathrm{RB}$ ). [To ensure that no auditory or visual cues were available during speech testing, subjects were blindfolded and exposed to masking noise.] 


\begin{tabular}{|c|c|c|c|c|c|c|c|}
\hline \multirow[b]{2}{*}{ Subject } & \multicolumn{2}{|c|}{ Isolated Syllables } & \multicolumn{2}{|c|}{ Isolated Words } & \multicolumn{2}{|c|}{ Sentences } & \multirow{2}{*}{$\begin{array}{l}\text { Connected-Discourse } \\
\text { Tracking } \\
\end{array}$} \\
\hline & 24 Consonants & 15 Vowels & Closed Se & Open Set & CID & IEEE & \\
\hline LD & $63 \%$ & $58 \%$ & $92 \%$ & $49 \%$ & $84 \%$ & $34 \%$ & 31 words/min \\
\hline $\mathrm{RB}$ & $58 \%$ & $46 \%$ & $72 \%$ & $34 \%$ & $85 \%$ & $47 \%$ & 30 words/min \\
\hline $\mathrm{JC}$ & $68 \%$ & $65 \%$ & $90 \%$ & $48 \%$ & $83 \%$ & $68 \%$ & 36 words/min \\
\hline
\end{tabular}

Table 1: Summary of speech reception measures with three Tadoma subjects. First column shows scores obtained in nonsense syllable tests using a set of 24 consonants in C-/a/ context and 15 vowels in /h/-V-/d/ context. Second column shows scores on isolated monosyllabic words for the five-alternative Modified Rhyme Test [11] and for phonetically balanced W-22 words [12]. Third column shows percentage correct identification of key words in lists of conversational CID sentences [13] and phonetically balanced IEEE [14] sentences for speaking rates of roughly 2.5 syllables/sec. Fourth column provides continuous-discourse tracking rate [15] in words/min.

\subsection{Speech-Reception Results}

The ability of Tadoma users to understand speech was examined for a variety of materials ranging from nonsense syllables to connected speech. In these studies, the Tadoma user placed his/her hand on the face and neck of the talker administering the test. For closed-response tests, the subject was provided with a list of the response alternatives in Braille and was asked to scan the list and select a response following each stimulus presentation. For open-response tests, the subject provided type-written or oral responses (which were clarified when necessary through spelling or fingerspelling).

Performance on a variety of speech tests is summarized in Table 1 for subjects $\mathrm{LD}, \mathrm{RB}$, and $\mathrm{JC}$.

At the basic segmental level, large sets of consonants or vowels are identified at a level of roughly $60 \%$ correct. Analyses of segmental errors indicate highly structured patterns of confusion that are quite similar across subjects. For consonants, confusions were concentrated on errors related to place of articulation within a given manner of production and also included confusions across several classes of production (e.g., affricates and fricatives, fricatives and semivowels); voicing confusions were rarely observed. Although vowel confusions were somewhat more broadly distributed than consonant confusions, three or four major clusters of errors were observed and related to misidentification of the properties of high-low and front-back positioning of the body of the tongue.

The role of linguistic context in the speech-reception abilities of these subjects is demonstrated by their scores on meaningful speech materials. The recognition rate for open-set words in isolation, for example, exceeds predictions based on performance on nonsensesyllables, but underestimates performance obtained on the reception of words in conversational sentences. These results imply that in openset recognition of isolated words the subjects were able to reject nonmeaningful responses through their semantic knowledge, and furthermore were able to exploit their knowledge of syntactical constraints in understanding words in conversational sentences. The superior performance observed on the CID compared to the IEEE sentences reflects differences in the amount of contextual information supplied by the two types of materials.
Such results are similar to those obtained in studies of the effects of speech-to-noise ratio on the auditory reception of speech materials as a function of context. A comparison of performance of Tadoma users on materials from the SPIN test [16] with that of normal-hearing listeners as a function of speech-to-noise ratio is presented in Fig. 1. In this test, the subject's task is to identify the final word in short sentences where the word is presented in either a high predictability (HP) or low predictability (LP) context. In Fig. 1, scores on HP and LP words reported by Kalikow et al. [16] for a group of older listeners (aged 60-75 yrs) are plotted as a function of speech-to-noise ratio. The scores achieved by each of the three Tadoma users are superimposed on the auditory data such that their HP scores are plotted on the HP



Figure 1. SPIN test results.

curve and their LP scores on the LP curve. The Tadoma scores range between $66-86 \%$ correct for HP words and between $30-44 \%$ for LP words. These scores correspond to performance achieved by the listeners at speech-to-noise ratios in the range of roughly $0-5 \mathrm{~dB}$. Additionally, the difference between HP and LP scores observed for the Tadoma users (32-56 percentage points) falls in the range of the maximal context effects observed for listeners in noise.

The effect of speaking rate on sentence intelligibility was examined by asking talkers to vary their subjective rate of production from slow to fast across different lists of sentences. A range of speaking rates from 
roughly 2-8 syllables/sec (where 5 syllables/sec is representative of normal speaking rate) was produced by the talkers administering these tests. In Fig. 2, the percent-correct reception of key words in lists of CID sentences is plotted as a function of speaking rate in syllables/sec for each of the three subjects. Performance falls off at rates above roughly 3-4 syllables/sec for RB and JC and 6 syllables/sec for LD. Across the sample of nine subjects tested in the survey study, however, maximal performance is typically obtained at rates roughly half those of the normal speaking rate [7].

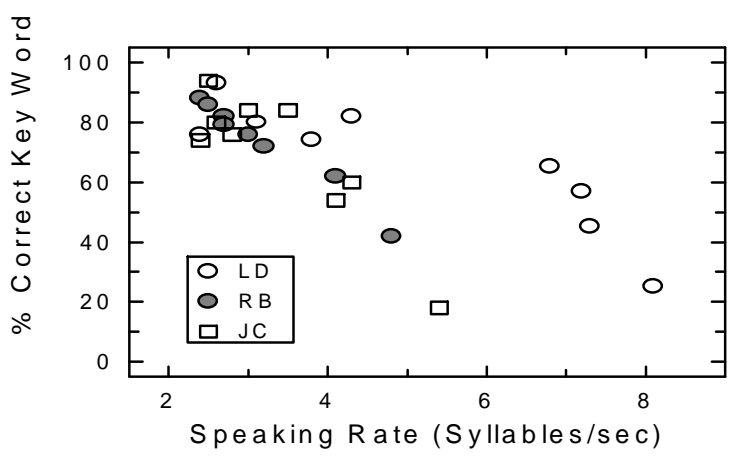

Figure 2. CID key-word reception as a function of rate.

The continuous-discourse tracking procedure [15] was employed to provide a practical estimate of the communication rates achieved by pairs of talkers and Tadoma users. For auditory reception of speech under normal conditions, tracking rate is roughly 100 words $/ \mathrm{min}$. The tracking rates attained by the Tadoma users (30-36 words/min) are roughly one-third as fast as the rates achieved in normal auditory reception of speech. These rates are limited primarily by the time spent correcting errors and secondarily by the slower speaking rates adopted by the talkers and the slower speaking rates employed by the Tadoma users [10].

\section{HOW DOES THE TADOMA USER PROCESS SPEECH?}

The reception of speech through Tadoma is based entirely on information concerning the various movements and actions that take place during articulation and that can be felt through the placement of the hand on the face and neck. The primary cues available to the Tadoma user include the up-down and in-out movements of the lips, movements of the jaw, airflow at the lips, and vibration on the neck. Insight into the relation between these cues and the reception of information concerning consonant and vowel segments has been derived from studies of segmental confusions made both by experienced Tadoma users [6,7,8] and laboratory-trained subjects [17] and from experiments conducted with an artificial Tadoma system $[18,19]$. For example, consonant voicing appears to be cued primarily by vibration that can be felt on both the neck and jaw and secondarily by airflow characterisitcs, while different manners of articulation are distinguished by differences in the intensity and concentration of airflow at the lips. The most salient information for vowels appears to be provided by in-out and up-down lip movements for determining roundedness and lip separation and by jaw movements for distinguishing vowels in which the body of the tongue is lowered. These cues appear to be sufficient for transmitting roughly 3 bits of information for consonants and 2 bits for vowels [6]. Incomplete segmental information appears to be combined with semantic and syntactic knowledge, leading to the ability to receive contextual messages with a reasonable degree of accuracy.

The uniqueness of successful Tadoma users lies in their ability to process continuous streams of tactual stimuli in such a way as to derive meaning from these patterns. While all the Tadoma users surveyed exhibited very similar performance at the segmental level, the distinguishing characteristic of the more successful users appears to lie in their ability to exploit the use of contextual cues to decode spoken messages [7]. Similarly, studies with naive laboratory subjects indicated that while they could be trained to identify speech segments with 50-100 hrs of practice [17], the goal of conversational speech reception comparable to that of experienced Tadoma users was not attained within 500-600 hrs of practice.

\section{IMPLICATIONS OF TADOMA FOR SENSORY SUBSTITUTION IN SPEECH COMMUNICATION}

The speech-reception abilities of the Tadoma users documented above demonstrate the capability of the tactual sense for supporting speech and language processing. For subject JC, for whom speech and language was already well-established at onset of deaf-blindness, the results presented here indicate her ability to substitute a tactual representation of the speech code for the auditory code with which she was previously familiar. For subjects LD and RB, the implications are somewhat more far-reaching. For these two individuals, the normal process for acquiring speech and language was interrupted in the early stages. Thus, their ability to receive speech and make use of contextual information requiring sophisticated linguistic knowledge indicates that tactual input served to establish a language base as well as to provide access to speech.

Careful scrutiny of the properties of the Tadoma method that account for its success may aid in the advancement of research on artificial tactual displays for speech communication. Of the various methods of encoding and displaying the speech signal to the tactual sense that have been studied in the development of artifical devices [e.g., see 20 and 21], none has resulted in performance through the tactual system alone comparable to that achieved through the Tadoma method. A combination of various factors may contribute to the success of the Tadoma method [discussed in 7 and 21, e.g.]. First, skilled users of this method have received intensive, long-term training and have employed the method over extended periods of time. Second, the multidimensional aspects of the speech display available through Tadoma appear to satisfy various principles of information theory for optimizing information transfer. Third, the use of the hand for reception of tactual stimulation may constitute an advantage from two points of view: the high density of innervation of tactual receptors and the potential engagement of both the cutaneous and kinesthetic components of the tactual sense. Finally, access to the articulators may constitute an advantage, especially in the view of proponents of a motor theory of speech perception. 
The current challenge in research on artificial tactual systems is the development of encoding and display schemes that will permit the reception of continuous speech at information-transfer rates at least as fast as those observed through Tadoma. Recent research has focused on improving information transfer through the development of tactual displays that engage the kinesthetic as well as the cutaneous component of that sensory system [see 22 and 23]. In a recent study exploring the

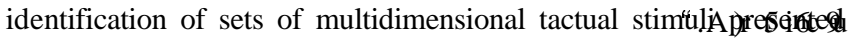
through a display capable of stimulation along the kinestheticcutaneous continuum, Tan [23] estimated information-transfer rates to be roughly $12 \mathrm{bits} / \mathrm{sec}$ (similar to that estimated for communication through Tadoma). Performance through the Tadoma method provides a standard for evaluating the performance of artificial tactual systems, as well as serving to demonstrate the potential of the tactual sense for the communication of speech and language.

\section{ACKNOWLEDGMENTS}

Work supported by research grant number 2 R01 DC 00126 from the National Institute on Deafness and Other Communication Disorders, National Institutes of Health.

\section{REFERENCES}

1. Alcorn, S. (1932). "The Tadoma Method," Volta Rev. 34: 195-198.

2. Stenquist, G. (1974). The Story of Leonard Dowdy: DeafBlindness Acquired in Infancy. Watertown, MA: Perkins School for the Blind.

3. Vivian, R. (1966). "The Tadoma Method: A Tactual Approach to Speech and Speech Reading," Volta Rev. 68: 733-737.

4. Schultz, M.C., Norton, S.J., Conway-Fithian, S., and Reed, C.M. (1984). "A Survey of the Use of the Tadoma Method in the United States and Canada," Volta Rev. 86: 282-292.

5. Norton, S.J., Schultz, M.C., Reed, C.M., Braida, L.D., Durlach, N.I., Rabinowitz, W.M., and Chomsky, C. (1977). "Analytic Study of the Tadoma Method: Background and Preliminary Results," J. Speech Hearing Res. 20: 574-595.

6. Reed, C.M., Durlach, N.I., Braida, L.D., and Schultz, M.C. (1982). "Analytic Study of the Tadoma Method: Identification of Consonants and Vowels by an Experienced Tadoma User," J. Speech Hearing Res. 25: 108-116.

7. Reed, C.M., Rabinowitz, W.M., Durlach, N.I., Braida, L.D., Conway-Fithian, S., and Schultz, M.C. (1985). "Research on theTadoma Method of Speech Communication," J. Acoust. Soc. Am. 77: 247-257.

8. Reed, C.M., Durlach, N.I., Braida, L.D., and Schultz, M.C. (1989). "Analytic Study of the Tadoma method: Effects of Hand Position on Segmental Speech Perception," J. Speech Hearing Res. 32: 921-929.

9. Chomsky, C. (1986). "Analytic Study of the Tadoma Method:
Language Abilities of Three Deaf-Blind Subjects," J. Speech Hearing Res. 29: 332-347.

10. Tamir, T.J. (1989). Characterization of the Speech of Tadoma Users. B.S. Thesis, MIT, Cambridge, MA.

11. House, A.S., Williams, C.E., Hecker, M.H.L., and Kryter, K.D. al $\mathrm{t}$ i o $\mathrm{n}$ T e s t in g M thods: C o n s o n a n t a 1 Differentiation with a Closed-Response Test," J. Acoust. Soc. Am. 37: $158-166$.

12. Hirsh, I.J., Davis, H., Silverman, S.R., Reynolds, E., Eldert, E., and Benson, R.W. (1952). "Development of Materials for Speech Audiometry," J. Speech Hearing Dis. 17: 321-337.

13. Davis, H., and Silverman, S.R. (1970). Hearing and Deafness. New York: Holt, Rhinehart, and Winston.

14. IEEE (1969). "Recommended Practice for Speech Quality Measurements," IEEE Transactions on Audio Electroacoustics 17: 225-246.

15. De Filippo, C.L., and Scott, B.L. (1978). "A Method for Training and Evaluating the Reception of Ongoing Speech.," J. Acoust. Soc. Am. 63: 1186-1192.

16. Kalikow, D.N., Stevens, K.N, and Elliott, L.L. (1977). "Development of Test of Speech Intelligibility in Noise using Sentence Materials with Controlled Word Predictability," J. Acoust. Soc. Am. 61: 1337-1351.

17. Reed, C.M., Doherty, M.J., Braida, L.D., and Durlach, N.I. (1982). "Analytic Study of the Tadoma Method: Further Experiments with Inexperienced Observers," J. Speech Hearing Res. 25: 216-223.

18. Leotta, D.F., Rabinowitz, W.M., Reed, C.M., and Durlach, N.I. (1988). "Preliminary Results of Speech-Reception Tests Obtained with the Synthetic Tadoma System," J. Rehab. Res. 25: 45-52.

19. Henderson, D.R. (1989). Tactile Speech Reception: Development and Evaluation of an Improved Synthetic Tadoma System. M.S. Thesis, MIT, Cambridge, MA.

20. Reed, C.M., Durlach, N.I., Delhorne, L.A., Rabinowitz, W.M., and Grant, K.W. (1989). "Research on Tactual Communication of Speech: Ideas, Issues, and Findings," Volta Rev. 91: 65-78.

21. Bernstein, L.E. (1992). "The Evaluation of Tactile Aids." In I.R. Summers (Ed.) Tactile Aids for the Hearing Impaired (pp. 167186). London: Whurr Publishers.

22. Eberhardt, S.P., Bernstein, L.E., Barac-Cikoja, D., Coulter, D.C., and Jordan, J. (1994). "Inducing Dynamic Haptic Perception by the Hand: System Description and Some Results," Proc. Am. Soc. Mech. Eng. 55: 345-351.

23. Tan, H.Z. (1996). Information Transmission with a Multi-Finger Tactual Display. Ph.D. Thesis, MIT, Cambridge, MA. 